\title{
Marker-assisted mapping enables effective forward genetic analysis in the arboviral vector Aedes aegypti, a species with vast recombination deserts
}

Chujia Chen ${ }^{1,2 *}$, Austin Compton ${ }^{2,3 *}$, Katerina Nikolouli * ${ }^{4}$ Aihua Wang ${ }^{2,3}$, Azadeh Aryan², $^{2}$, Atashi Sharma ${ }^{2,3}$, Yumin $\mathrm{Qi}^{2,3}$, Camden Delinger, ${ }^{2,3}$, Melanie Hempel ${ }^{2,3}$, Antonios Augustinos ${ }^{4,5}$, David W. Severson ${ }^{6}$, Kostas Bourtzis ${ }^{4}$ and Zhijian Tu ${ }^{1,2} 3 \S$

1. Genetics Bioinformatics and Computational Biology Program, Virginia Tech, Blacksburg, VA24061, USA

2. Fralin Life Sciences Institute, Virginia Tech, Blacksburg, VA24061, USA

3. Department of Biochemistry, Virginia Tech, Blacksburg, VA24061, USA

4. Insect Pest Control Laboratory, Joint FAO/IAEA Centre of Nuclear Techniques in Food and Agriculture, Department of Nuclear Sciences and Applications, IAEA Laboratories, 2444 Seibersdorf, Austria

5. Present address: Hellenic Agricultural Organization-Demeter, Institute of Industrial and Forage Crops, Department of Plant Protection Patras, Patras, 26442, Greece

6. Department of Biological Sciences, University of Notre Dame, Notre Dame, IN 46556

* Equal contribution

§ Please send correspondence to: Zhijian Jake Tu (jaketu@vt.edu) Or Kostas Bourtzis (K.Bourtzis@iaea.org) 


\begin{abstract}
Aedes aegypti is a major vector of arboviruses that cause dengue, chikungunya, yellow fever and Zika. Although recent success in reverse genetics has facilitated rapid progress in basic and applied research, integration of forward genetics with modern technologies remains challenging in this important species, as up-to- $47 \%$ of its chromosome is refractory to genetic mapping due to extremely low rate of recombination. Here we report the development of a marker-assistedmapping (MAM) strategy to readily screen for and genotype only the rare but informative recombinants, drastically increasing both the resolution and signal-to-noise ratio. Using MAM, we mapped a transgene that was inserted in a $>100 \mathrm{Mb}$ recombination desert and a sex-linked spontaneous red-eye (re) mutation just outside the region. We subsequently determined, by CRISPR/Cas9-mediated knockout, that cardinal is the causal gene of re, which is the first forward genetic identification of a causal gene in Ae. aegypti. This study provides the molecular foundation for using gene-editing to develop versatile and stable genetic sexing methods by improving upon the current re-based genetic sexing strains. MAM does not require densely populated markers and can be readily applied throughout the genome to facilitate the mapping of genes responsible for insecticide- and viral-resistance. By enabling effective forward genetic analysis, MAM bridges a significant gap in establishing Ae. aegypti as a model system for research in vector biology. As large regions of suppressed recombination are also common in other plant and animal species including those of economic significance, MAM will have broad applications beyond vector biology.
\end{abstract}




\section{Introduction}

Aedes aegypti is a major vector of a number of arboviruses and filarial worms. Effective control of this one species could help reduce or prevent a number of vector-borne infectious diseases including dengue, chikungunya, yellow fever and Zika. Current strategies to reduce the incidence and burden of these diseases depend heavily on effective vector control, which is hindered by increasing insecticide-resistance. Novel control strategies, informed by improved understanding of mosquito biology, are urgently needed. Recent years witnessed rapid accumulation of genomic resources (e.g., (Matthews et al. 2018)) and effective applications of CRISPR/Cas9-mediated reverse genetic analysis of gene function in Ae. aegypti (e.g., (Hall et al. 2015); (Li et al. 2017); (Aryan et al. 2020)). However, successful integration of forward genetics with modern technologies to identify causal genes of important traits or phenotypes, as championed by many plant and animal geneticists (e.g. (Schneeberger 2014); (Navarro-Escalante et al. 2020); (Feng et al. 2021)), still awaits in this important vector species.

Recombination is fundamental to forward genetic screening and quantitative trait loci (QTL) mapping. Mapping a locus that contains the causal gene is possible when recombination separates the causal gene locus from its neighboring sequences, resulting in an enrichment of markers tightly linked to the causal gene in individuals manifesting the phenotype (Figure 1). However, Ae. aegypti has a lower per megabase recombination rate $(\sim 0.3 \mathrm{cM} / \mathrm{Mb})$ than Anopheles gambiae (0.8 cM/Mb) and Drosophila melanogaster (1.6 cM/Mb) (Wilfert, Gadau, and Schmid-Hempel 2007). In addition, up to $47 \%$ of its chromosome surrounding the centromere belongs to so-called low recombination regions (LRRs), with rates much lower than the $0.3 \mathrm{cM} / \mathrm{Mb}$ average ((Juneja et al. 2014); (Fontaine et al. 2017); (Dudchenko et al. 2017)). Although it is not unique to Ae. aegypti to have LRR near centromeres, having nearly half of the genome in LRR further complicates genetic efforts to map genes that determine particular traits. Thus, the low recombination rate in Ae. aegypti limits both the resolution and efficiency of these genetic mapping studies. We are not aware of any report of the successful determination of a causal gene using forward genetics in Ae. aegypti, despite the intense interest in identifying genes that underly insecticide- and pathogen-resistance or genes that could be used as selectable markers for genetic sexing (Koskinioti et al. 2021; Ward et al. 2021). The presence of extensive regions with very low recombination rates, or recombination deserts, has also been reported in many plant and animal species (Stapley et al. 2017a, 2017b). Therefore, forward genetic mapping in these recombination deserts is a significant and broadly important challenge.

Here we report the development of a marker-assisted-mapping (MAM) strategy and demonstrate its success in rapidly mapping a transgene insertion in a region of suppressed recombination near the sex locus in Ae. aegypti. Using the same method in conjunction with introgression analysis, we also identified the causal gene for a spontaneous red-eye (re) mutation in Ae. aegypti, which was first reported nearly 60 years ago (McClelland 1962; McClelland 1966). The re mutation is currently used for developing genetic sexing strains (GSSs) due to its sex-linkage and the robustness of the re strain (Koskinioti et al. 2021). This study provides the molecular foundation for future improvements and expansion of GSSs and enables effective forward genetic analysis in Aedes aegypti. As large regions of suppressed recombination are also common in other plant and animal species including those of economic significance, MAM will have broad applications beyond vector biology.

\section{RESULTS}

The marker-assisted mapping (MAM) strategy. The general strategy of MAM and its advantages are depicted in Figure $1 \mathrm{~A}$ and $1 \mathrm{~B}$, respectively. Rare and informative recombinants are selected for genotyping analysis based on the breakage of linkage of the selectable markers 
( $A$ and $B$ ) flanking the target locus ( $T$ or $t$ ), thus greatly increasing both the resolution and efficiency of genetic mapping. In the example shown in Figure 1, 99\% of the non-informative progeny are ignored, thus both the resolution and signal-to-noise-ratio are increased by 100 fold. If informative positive samples and informative negative samples are genotyped in bulk, MAM represents a novel application of the bulk-segregant analysis (Schneeberger 2014) that can identify causal genes in previously inaccessible regions of suppressed recombination.

The chromosomal region under investigation and the markers. The low recombination regions (LRRs) in Ae. aegypti (Dudchenko et al. 2017), which encompass up to $47 \%$ of a chromosome, show little or no recombination at the resolution of the available genetic crosses (Juneja et al. 2014). The same pattern of vast regions of suppressed recombination is reproduced (Supplemental Figure S1) using the most recent PacBio-based assembly (Matthews et al. 2018). We recently obtained a sex-linked transgenic insertion line named P10 (Supplemental Figure S2, Table 1), which expresses a polyUb-driven GFP. The transgene is inserted at position $224.7 \mathrm{Mb}$ of the $\mathrm{q}$ arm of chromosome 1, as shown by Oxford Nanopore sequencing (Supplemental Figure S3). The genetic distance between P10 and the sex locus (M locus, 151.68-152.95 Mb on chromosome 1) is approximately 1.02 to $1.55 \mathrm{cM}$ (Table 1). Thus, the per megabase recombination rate in the $\sim 72 \mathrm{Mb}(224.68-152.95=71.73 \mathrm{Mb})$ region is $0.014-0.022 \mathrm{cM} / \mathrm{Mbp}$, approximately 73 to 114 fold lower than that of the $D$. melanogaster $(1.6 \mathrm{cM} / \mathrm{Mb})$ (Wilfert, Gadau, and Schmid-Hempel 2007). A previously reported recessive red-eye (re) locus is also on the $q$ arm of chromosome 1 , and it is 2-3 cM away from the M-locus, with an unknown chromosomal location (Augustinos et al. 2020; Koskinioti et al. 2021). We first seek to establish the markerassisted mapping (MAM) strategy by mapping the location of the P10 insertion using the maleness (M locus) and wildtype black-eye (+/+ or $+/$ re) as flanking markers (Figure 2).

Rapid and effective mapping of the P10 transgene using marker-assisted mapping. To map P10 which expresses GFP, rare and informative recombinants are selected for genotyping analysis based on the breakage of linkage between the two flanking markers, maleness and black-eye. A backcross (Figure 2) produced dozens of informative recombinants among thousands of non-informative progeny. We grouped the informative recombinants into four categories for Illumina sequencing (Supplemental Table S1) as we are also interested in mapping $r e$ in a later analysis (see below). The four groups include male positive red-eye (MPR); female positive black-eye (FPB); female negative black-eye (FNB); male negative red-eye (MNR). As shown in Figure 3, when MPR and FPB (all informative P10-positive recombinants) are compared with FNB and MNR (all informative P10-negative recombinants), MAM successfully mapped the P10 location to be between 223.3 and $226.1 \mathrm{Mb}$ with $95 \%$ confidence. The midpoint of the peak is $224.7 \mathrm{Mb}$, precisely the location of the P10 insertion.

Marker-assisted mapping of re. The above-mentioned sequencing data are also used to map re, as we can compare the recombinant male red-eye and the recombinant female black-eye samples. A potential caveat is the lack of resolution from the telomeric side of $r e$, as the cross was originally intended for mapping P10 and a marker to the telomeric side of re was not used. However, given the interest in developing re as a marker for GSS (Koskinioti et al. 2021), the fact that $r e$ is one of the earliest reported spontaneous mutations isolated in Ae. aegypti (McClelland 1966; McClelland 1962), and the lack of any successful examples of forward genetic identification of causal genes in Ae. aegypti, we attempted to map re using the existing data. As shown in Figure $4 \mathrm{~A}$, the re locus is mapped to the region between $271 \mathrm{Mbp}$ and $278 \mathrm{Mbp}$ with $95 \%$ confidence.

Mapping re using the introgression lines. The above mapping result for re is unexpected as the previously published data indicate that the genetic distance between the sex locus and the 
271-278 Mb region is at least $10 \mathrm{cM}$ (Dudchenko et al. 2017), much higher than the $2.48 \mathrm{cM}$ observed between the sex locus and re (Figure 2 legend). We sought independent evidence for the location of re. As a part of an ongoing effort to develop GSS, the Rexville red-eye strain (Koskinioti et al. 2021) was introgressed with Ae. aegypti from various regions for a minimum of 11 generations to establish GSSs of various local genetic backgrounds (Supplemental methods). The re-containing strain used in the above-mentioned MAM experiment is the RED strain, which is homozygous for the re locus on chromosome 1, the $s$ (spot abdomen) locus on chromosome 2 , and the b/t (black tarsus) on chromosome 3 (Craig and Hickey 1967). Genetic complementation crosses confirmed that the re in RED and the red-eye locus in the Rexville-derived introgression lines share the same mutant gene (Supplemental Figure S4). These introgressed GSSs contain the re mutation in divergent genetic backgrounds, which could be used to narrow down the location of re, by identifying regions of enriched homozygosity. To increase the cost-effectiveness, we performed Illumina sequencing of red-eyed hybrids (Supplemental Table S1) from crosses between different GSSs to further highlight the enrichment of homozygosity near the re locus. As shown in Figure 4B-D, homozygosity is the highest in a region around 271-272 Mb. We note that the homozygosity peak of the three red-eyed introgression samples falls on the centromeric side of the MAM peak (271-278 Mb), which is consistent with expectation as the P10 marker is to the centromeric side of $r e$ and there is no marker to the telomeric side of $r e$.

Identification of a candidate gene cardinal. To further narrow down the candidate region, we focused the homozygosity analysis on the $265-280 \mathrm{Mb}$ region using combined SNP data from all three hybrid red-eyed samples. As shown in Figure 5, the region with the highest homozygosity is around $271.5 \mathrm{Mb}$. Interestingly, a gene encoding a chorion peroxidase, which is an ortholog of the Drosophila eye pigment gene cardinal, is located between 271.574 and $271.583 \mathrm{Mb}$ and this gene is expressed throughout development after $20 \mathrm{hr}$ post egg deposition and its transcripts are enriched in the brain of both sexes in Ae. aegypti (Matthews et al. 2018). Thus, cardinal is the top candidate for the causal gene for the spontaneous re mutation. Indeed, the cardinal gene sequences in red-eyed samples show a number of non-synonymous mutations leading to changes of amino acid residues that are conserved across divergent mosquitoes (Supplemental Figure S5).

CRISPR/Cas9-mediated knockout of cardinal produced somatic knockouts showing mosaic red-eyed $G_{0}$ individuals. To increase the likelihood to observe a somatic cardinal knockout phenotype in the $\mathrm{G}_{0}$, we first performed CRISPR/Cas9-mediated knockout in heterozygous +/re individuals. The sgRNAs used to target cardinal are shown in Figure 6A and provided in Supplemental Table S2. Mosaic red-eyes were indeed observed in $+/ r e \mathrm{G}_{0}$ individuals (Figure 6B; Supplemental Figure S6). Such somatic mosaicism may reflect knockout of the wildtype cardinal allele (cd-) in some cells, resulting in the red-eye phenotype in the cd-/re genotyped cells. Thus, the result is consistent with cardinal being the causal gene of the re mutation. However, we cannot rule out the possibility that the mosaic phenotype may have nothing to do with re and it simply resulted from somatic knockout of both copies of the cardinal gene. Regardless, this result confirmed that the sgRNAs are effective and the cardinal gene is indeed involved in eye-color in Ae. aegypti, as recently demonstrated in Culex quinquefasciatus (Feng et al. 2020).

Germline knockout of cardinal showed that it is the causal gene of the re mutant. To isolate germline knockout of cardinal (Figure 6C), sgRNAs and Cas9 mRNAs were injected in embryos collected from wildtype Lvp females (+/+) mated with wildtype Lvp males (+/+). The resulting $\mathrm{G}_{0}$ females were black-eyed and selected to mate with red-eyed males (re/re). Four female and seven male red-eyed $\mathrm{G}_{1}$ individuals were identified among approximately 500 black-eyed $\mathrm{G}_{1}$ individuals (Supplemental Table S3; Figure 6C-D). PCR and subsequent Sanger sequencing of 
5 red-eyed $\mathrm{G}_{1}$ males using primers flanking the sgRNAs (Supplemental Table S4) identified two types of mutations. A single base deletion that results in a frameshift was found in 4 of the $\mathrm{G}_{1}$ males (\#1, 5, 6, and 7) and a 99 base deletion in the other $\left(\mathrm{G}_{1}\right.$ male \#3; Figure $\left.6 \mathrm{E}\right)$. However, none of these deletions were found in the black-eyed $\mathrm{G}_{1}$ individuals (Figure $6 \mathrm{E}$ ). These red-eyed $\mathrm{G}_{1}$ individuals can only have one re allele from the father and one cd- allele from the mother. Therefore, cardinal knockouts (cd-) resulting from either 1- or 99-bp deletion fail to complement re, suggesting that cardinal is indeed the causal gene of the re mutant.

Establishing and characterizing a homozygous cardinal knockout. To obtain homozygous knockout individuals, black-eye $\mathrm{G}_{2}$ females (+/cd-) were backcrossed to their father, red-eye $\mathrm{G}_{1}$ male\#3 (also +/cd-; Figure 7A). Their $\mathrm{G}_{3}$ offspring were evaluated and four genotypes were expected and four phenotypes were observed: red-eyed males, black-eyed males, yellow-eyed females, and black-eyed females (Figure 7B, Supplemental Table S5). The yellow-eyed females are homozygous for the $99 \mathrm{bp}$ cardinal deletion. The yellow-eyed phenotype is observed in the pre-pupal compound eye (Figure 7B and Supplemental Figure S7). However, the yellow-eyes gradually turned into red-eyes over the course of pupae development and adult emergence (Figure 7C-D). Therefore, the yellow-eye phenotype is in fact yellow-turn-red. No yellow-eyed L2/L3 instars were found and the ocelli of the yellow-eyed pre-pupa are red, indicating that the yellow-turn-red phenotype is associated with the remodeling of the compound eye during metamorphosis. The sibling red-eyed males inherited one copy of the re spontaneous mutation and one copy of the cardinal knockout (Figure 7A), again indicating that $c d$-does not complement re. As expected, when the $\mathrm{G}_{2}$ females (+/cd-) were crossed to RED males ( $\mathrm{re} / \mathrm{re}$ ), approximately half of the offspring are red-eyed in each sex (Supplemental Figure S8 and Table S6).

\section{DISCUSSION}

Marker-assisted mapping (MAM) enables effective forward genetic analysis in and outside the recombination desert in Ae. aegypti. There has not been a report of a successful forward genetic discovery of the causal gene of any mutant phenotype in Ae. aegypti, despite the significant interest in the fundamental biology and vectorial capacity of this key arboviral vector. The overall low recombination rate $(0.3 \mathrm{cM} / \mathrm{Mb}$, (Wilfert, Gadau, and Schmid-Hempel 2007)) and the existence of vast recombination deserts known as the LRRs ((Juneja et al. 2014); (Fontaine et al. 2017); (Dudchenko et al. 2017); Supplemental Figure S1) represent a key bottleneck for forward genetics in Ae. aegypti. We designed the marker-assisted mapping strategy to overcome this key bottleneck by selecting and genotyping only the rare but informative recombinants between easy-to-screen markers flanking the target locus, thus, drastically improving the resolution, signal-to-noise ratio, and cost-effectiveness for genetic mapping (Figure 1). Using MAM, we successfully mapped P10, a dominant transgene insertion that shows an extremely low rate of recombination relative to the sex locus $(0.014-0.022 \mathrm{cM} / \mathrm{Mbp})$ and the red-eye (re) marker (1.46 cM/46 Mb=0.032 cM/Mb). The MAM peak spans from 223.3 Mb to $226.1 \mathrm{Mb}$ with the midpoint being $224.7 \mathrm{Mb}$, precisely the location of the P10 insertion. Therefore, we have demonstrated the power of MAM in making forward genetics accessible in the LRRs which were previously inaccessible or challenging.

The same set of MAM data was also used to map re, a recessive spontaneous mutant. Despite the lack of a marker to the telomeric side of $r e$, we were able to map re to a broader peak between $271.1 \mathrm{Mb}$ and $277.8 \mathrm{Mb}$ at a $95 \%$ confidence interval. The location of the re causal gene cardinal is at $271.5 \mathrm{Mb}$, much closer to the centromeric side $(271.1 \mathrm{Mb})$ of the peak than to the telomeric side. The lack of resolution at the telomeric side, as indicated by the $6.3 \mathrm{Mb}$ distance between $271.5 \mathrm{Mb}$ and $277.8 \mathrm{Mb}$, likely reflects the lack of a marker to "assist" in selecting informative recombinants. The resolution from the telomeric side could be even worse if the telomeric side of 
re were in the LRR. We also showed that homozygosity analysis using hybrids between repeatedly introgressed strains can also facilitate mapping studies. The introgressed GSSs are highly valuable but time consuming to create as a minimum of 11 generations were required, thus may not be available to other mapping studies. However, even in the absence of one of the two flanking markers and without the use of additional resources such as these introgression lines, MAM can still identify the candidate locus with the causal gene being proximal to the marker-side of the peak.

As mentioned earlier, the location of $r e$ is somewhat unexpected as the previously published data indicate that the genetic distance between the sex locus and the $271.5 \mathrm{Mb}$ region is at least 10 cM (Dudchenko et al. 2017), much higher than the 2-3 cM between the sex locus and re (Augustinos et al. 2020; Koskinioti et al. 2021 and Figure 2 legend). Although such a discrepancy may result from different genetic backgrounds or other conditions that may affect the rate of recombination (Craig and Hickey 1967), it may also reflect a limitation of the previous mapping studies in which a small number of progenies were genotyped. The ease of screening for the P10 and re markers affords a high-resolution estimate of the recombination rate based on rapid screening of a large number of progenies. This is especially important when working with loci in the LRRs.

The identification of cardinal as the causal gene for the spontaneous re mutant is the first report of a successful identification of the causal gene using forward genetics in Ae. aegypti. The cardinal gene is outside the previously defined LRRs ((Juneja et al. 2014); (Fontaine et al. 2017); (Dudchenko et al. 2017)). Thus, we have shown that MAM enabled forward genetics both in and outside the LRRs. Our study focused on the 1q arm which is approximately $159 \mathrm{Mb}$ long, spanning from the centromeric sex locus (151.68-152.95 Mb) to the telomere (located at $311 \mathrm{Mb}$ ). P10 (located at 224.7 Mb) and re (located at 271.5 Mb) are $72 \mathrm{Mb}$ and 119.5 Mb away from the sex locus, respectively. Therefore, it is not necessary to have densely populated markers for MAM to succeed. With the ease in obtaining transgene insertions and the availability of targeted knock-in (Kistler, Vosshall, and Matthews 2015), MAM can be readily established throughout the entire Ae. aegypti genome to greatly facilitate forward genetic studies. Knocking in a marker near a candidate gene can also help locate the precise location of the causal gene before embarking on functional analysis (Ding et al. 2016). Coupling MAM with targeted genotyping, in place of wholegenome sequencing, will further improve the efficiency and reduce the cost of genetic mapping efforts.

Insights into the function of cardinal. We did not observe any phenotypic differences between $\mathrm{re} / \mathrm{re}$ and $\mathrm{re} / \mathrm{cd}$ - individuals. However, the homozygous progeny of a 99 bp deletion in cardinal showed a phenotype of eyes gradually turning from yellow to red. The yellow-eyed phenotype was first observed in the pre-pupal compound eye and gradually turned into red-eyes over the course of pupae development and adult emergence. Thus, the phenotype is associated with the remodeling of the compound eye during metamorphosis. The cardinal gene encodes a peroxidase which catalyzes the formation of the eye pigment xanthommatin from 3-hydroxykynurenine in the ommochrome pathway and is believed to also participate in the synthesis of ommin ((Howells, Summers, and Ryall 1977); (Harris et al. 2011); (Osanai-Futahashi et al. 2016); (Zhang et al. 2017); (Xu et al. 2020)). Indeed, knockout of cardinal near its C-terminus in Culex quiquefasciatus resulted in a red-eye mutation visible from the larval to the early adult (Feng et al. 2020), while an amorphic cardinalknockout in the diamond back moth resulted in yellow-eyed adults which turned into red-eyed as they aged (Xu et al. 2020). The gradual change from yellow to red in eye color was explained by the slow auto-oxidation of the yellow pigment 3-hydroxykynurenine leading to the eventual accumulation of the red pigment xanthommatin. Similarly, the $99 \mathrm{bp}$ cardinal deletion 
in Ae. aegypti likely resulted in either an amorphic mutation or a more severe hypomorphic mutation than the spontaneous re.

Implications to the development of GSSs. The development of GSSs has been a key challenge to SIT programs from the start. Indeed, it took more than 20 years to develop one of the most successful GSSs for the Mediterranean fruit fly, the Ceratitis capitata VIENNA 8 GSS (Franz et al. 2021). Only recently the causal gene of the white-pupae (wp) mutation used in the VIENNA 8 GSS was identified (Ward et al. 2021). The necessity to develop a mosquito GSS does not only stem from economic concerns but also from the behavioral traits of the species since only females feed on vertebrate hosts and transmit pathogens. Therefore, GSS that allows for effective sexseparation is a critical component of any genetic approaches to control mosquito-borne infectious diseases. Several mosquito GSSs were developed for Anopheles species since the 1970s (Kaiser P.E. 1978, Robinson AS 1986, Curtis CF 1978, Lines JD 1985, Yamada et al. 2012, 2015). However, the high genetic instability of these strains and in some cases the reliance on insecticide-resistance as a selection marker are problematic. Recent field releases of transgenic, irradiated and/or Wobachia-infected sterile males showed promising results for suppressing Aedes populations in areas of moderate sizes (reviewed in (Papathanos et al. 2018), Kittayapong et al. 2019, (Zheng et al. 2019), (Crawford et al. 2020), Balatsos et al. 2021). However, robust, precise, and economical sex-separation remains a significant bottleneck to large-scale implementations of genetic measures to control mosquito-borne infectious diseases.

The current red-eye GSS has shown excellent performance and genetic stability under laboratory conditions (Koskinioti et al. 2021) and an induced chromosomal inversion further increased the precision and effectiveness of these GSS lines (Augustinos et al. 2020). In this study we identified the causal gene of the red-eye phenotype. The identification of the red-eye causal gene will facilitate the development of GSS lines, not only for Ae. aegypti but also for other medically important mosquitoes such as Ae. albopictus and Anopheles species. For example, the red-eye phenotype can be created by knocking out cardinal in these species using CRISPR/Cas-mediated genome editing approaches. Tight sex-linkage could be achieved either by X-ray or CRISPR/Cas9-induced chromosomal translocation, or by CRISPR/Cas9-mediated knock-in of a wildtype cardinal in the male sex locus. This would significantly accelerate the process of the GSS development thus saving time and resources.

Concluding remarks. Genomic, transcriptomic and population genomic resources have rapidly accumulated in the past few years for Ae. aegypti (e.g., (Matthews et al. 2018); (Mysore, Li, and Duman-Scheel 2018); (Rose et al. 2020)). Effective application of genome-editing and improved efficiency of transposon-mediated transformation ((Hall et al. 2015); (Li et al. 2017); (Aryan et al. 2020)) make Ae. aegypti increasingly amenable to genetic research. We anticipate that MAM will significantly improve forward genetic studies in Ae. aegypti and facilitate the mapping of genes responsible for insecticide- and viral-resistance, and/or other large-effect loci identified from QTL analyses. In addition to overcoming the challenges of mapping in the recombination deserts, MAM offers another advantage as time-consuming assays for insecticide- or viral-resistance will only need to be performed on informative recombinants. MAM fills a gap in establishing Ae. aegypti as a model system for basic and applied research in vector-borne infectious diseases. As large regions of suppressed recombination are also common in other plant and animal species including those of economic significance (Stapley et al. 2017a, 2017b), MAM will have broad applications beyond vector biology.

\section{Materials and Methods}


Mosquito rearing: The Liverpool (LVP) strain (obtained from www.BEl.org) and the RED strain (obtained from the Severson laboratory at the University of Notre Dame) of Ae. aegypti were maintained at Virginia Tech at $26-28^{\circ} \mathrm{C}$ and $60-70 \%$ relative humidity with a $14 / 10$ hour day/night light cycle. Adult mosquitoes were fed $10 \%$ sucrose and fed blood using artificial membrane feeders and defibrinated sheep's blood (Colorado Serum Company; Denver, CO).

The P10 Transgenic line: A piggyBac donor plasmid (500ng/ul, Supplemental Figure S2) that contains an EGFP transformation marker driven by the Ae. aegypti polyubiquitin promoter and was co-injected with an in vitro transcribed piggyBac mRNA (300ng/ul) into less than $1 \mathrm{hr}$ old embryos of the Liverpool strain of Ae. aegypti (Coates et al. 1998). The piggyBac-hsp70transposase (Handler et al. 1998) was used as a template for in vitro transcription using the mMessage mMachine T7 Ultra kit (Thermofisher), followed by MEGAclear (Thermofisher) column purification. Approximately 1200 embryos were injected, resulting in $150 \mathrm{G}_{0}$ females and $160 \mathrm{G}_{0}$ males). Surviving $\mathrm{G}_{0}$ females were mated to Liverpool males in pools of $20-25$. Each $\mathrm{G}_{0}$ male was mated individually with 5 Liverpool females in individual cages and mosquitoes from 15-20 of these cages were merged into one large pool. $\mathrm{G}_{1}$ larvae were screened for green fluorescence using a Leica M165 FC fluorescence microscope. Positive $G_{1}$ individuals were out-crossed to Liverpool mosquitoes to ensure that all transgene cassettes were stably inherited to the $\mathrm{G}_{2}$ generation.

Identification of the P10 insertion site: Oxford Nanopore sequencing was performed using genomic DNA isolated from four $\mathrm{G}_{5}$ P10-positive males. The Qiagen Genomic Tip DNA Isolation kit (Cat. No. 10,243 and 19,060, Qiagen, Hilden, Germany) was used. These four males were homogenized using a hand held pestle motor mixer (Cole Palmer) with a sterile disposable plastic pestle (USA Scientific) for approximately 10 seconds in the lysis buffer. The homogenate was incubated with $300 \mathrm{mAU}$ Proteinase K (Qiagen, Hilden, Germany) overnight at $55^{\circ} \mathrm{C}$, and then transferred into a $15 \mathrm{~mL}$ conical tube for centrifugation at $5000 \mathrm{~g}$ for 15 minutes at $4{ }^{\circ} \mathrm{C}$ to remove debris. The lysate was loaded on a $20-\mathrm{G}$ column for purification following the protocol. The purity, approximate size, and concentration of the DNA were measured using a nanodrop spectrophotometer, $0.5 \%$ agarose gel electrophoresis, and Qubit dsDNA assay, respectively. Approximately $2000 \mathrm{ng}$ gDNA was used to prepare the library using the 1D ligation sequencing kit (SQK-LSK108, Oxford Nanopore Technologies, UK). Adapter ligation was performed at room temperature for 45 minutes. Approximately $530 \mathrm{ng}$ of the prepared library was loaded on the flow cell. Approximately 7.6 Gbases reads were obtained and submitted to NCBI ((PRJNA718905, sample SAMN18579040). Four long reads that contain the piggyBac insertion were identified and used to map the location of the P10 insertion (Supplemental methods 1; Supplemental Figure S3).

Sample Collections for Marker assisted mapping: The crossing scheme is shown in Figure 2. GFP-positive, black-eyed P10 males $(n=66)$ were mated with GFP-negative, red-eyed females $(n=37)$ to generate the F1 progeny. The GFP-positive, black-eyed F1 males $(n=26)$ were backcrossed with GFP-negative, red-eyed females $(n=46)$ to generate F2 progeny. The F2 progenies were screened and counted. The following numbers of individuals were pooled for genomic DNA isolation and Illumina sequencing: male positive black-eyed $(n=60)$, male positive red-eyed $(n=12)$, female positive black-eyed $(n=20)$, female negative red-eyed $(n=60)$, male negative red-eyed $(n=11)$ and female positive black-eyed $(n=7)$. Illumina sequencing coverage and other statistics are provided in Supplemental Table S1.

MAM data analysis: Adapters and low quality Illumina reads were trimmed by Trimmomatic v0.38 (Bolger, Lohse, and Usadel 2014) and quality control were performed using FastQC v0.11.8. [https://www.bioinformatics.babraham.ac.uk/projects/fastqc/]. Variants were called using GATK 
v4.1 (McKenna et al. 2010) following the "Best Practices workflow" ((DePristo et al. 2011); (Van der Auwera et al. 2013)). First, paired reads of each sample were aligned to the Aedes aegypti genome L5 from VectorBase [www.vectorbase.org] using BWA-MEM v0.7.17 (Li and Durbin 2009) and sam files were converted to bam files and sorted by samtools (Li et al. 2009). PCR duplicates were marked by GATK. SNPs of each sample were called by GATK using the Haplotype caller mode. GVCF files were combined by GATK CombineGVCFs. Combined GVCF files were converted to VCF files by GATK GenotypeGVCFs. Variants are selected from VCF to a table by GATK VariantsToTable for the subsequent Bulk Segregant Analysis by QTLseqr (Takagi et al. 2013; Mansfeld and Grumet, 2018). Variants were filtered using GATK. The number of SNPs, minimum read depth, and tricube-smoothed $\Delta$ (SNP-index) within a $1 \mathrm{Mb}$ sliding window were calculated by QTLseqr. The slope of $\Delta$ (SNP-index) was used to calculate the inflection point. Simulations with 10000 bootstrap replicates were used to calculate the two-sided confidence intervals. The simulation was performed based on data derived from read depths and the type and size of the population. Alternative allele frequency was calculated per bulk and $\Delta$ (SNP-index) was calculated over multiple replications for each bulk under the null hypothesis that no QTLS exist. The percentile from the simulation was used to estimate the confidence intervals.

To map the P10 insertion, male negative red-eye (MNR) and female negative black-eye (FNB) were used as the control while the female positive black-eye (FPB) and male positive red-eye (MPR) were used as the experimental group. Variants were filtered based on $10<$ total read depth $<400,0.2<=$ sum of reference allele frequency of two bulks $<=0.8$, per sample read depth $>=5$ and GATK GQ score >= 99. Approximately 2.7 million SNPs remained after variant filtering. To map the re locus, female positive black-eye (FPB) and female negative black-eye (FNB) were used as the control while male negative red-eye (MNR) and male positive red-eye (MPR) were used as the experimental group. Variants were filtered based on the same parameters as mentioned above. Approximately 2.5 million SNPs remained after variant filtering.

Ae. aegypti strains used for homozygosity analysis. All strains described in this section were maintained in the insectary of the Insect Pest Control Laboratory (Joint FAO/IAEA Center of Nuclear Techniques in Food and Agriculture, Seibersdorf, Austria) at $27 \pm 1{ }^{\circ} \mathrm{C}, 80 \%$ relative humidity and a 12/12 h day/night photoperiod. Adult mosquitoes were provided a $10 \%$ sucrose solution and females were fed with porcine blood twice per week. The blood used was collected in Vienna, Austria during routine slaughtering of pigs in a nationally authorized abattoir, conducted at the highest possible standards strictly following EU laws and regulations. Egg collections were initiated $72 \mathrm{~h}$ after the last blood feeding using moistened oviposition papers (white germination paper, Sartorius Stedium Biotech, Austria). The Rexvillle strain was provided by Dr. Margareth Capurro at the Department of Parasitology, University of Sao Paulo, Brazil. All Rexvillle individuals have red eye color which is evident throughout all developmental stages and darkens as adults age. The Rexville strain has been shown to complement the red-eye mutation (re) in the RED strain (Supplemental Figure 4). The Rexville red-eye phenotype was introgressed into the BRA, Mex, Thai, and Pak strains to create red-eye genetic sexing strains (GSSs) with different genomic backgrounds in a separate study (Augustinos et al. 2020; Koskinioti et al. 2021). The 11generation introgression protocol is described in Supplemental Methods 2.

Crosses and sample preparation for the homozygosity analysis. The above mentioned introgressed GSS contain the re mutation in divergent genetic backgrounds, which could be used to narrow down the location of re, by identifying regions of enriched homozygosity. Thirty F8 redeye females from the THAI Red-Eye GSS and fifteen F9 red-eye males from the MEX Red-Eye GSS were mass-crossed. All progeny was red-eyed and a pool of 20 freshly emerged adult 
females (F8ThaiF9Mex_F) and a pool of 20 males (F8ThaiF9Mex_M) were used to extract DNA using the QIAGEN Genomic tip 20/G kit (Qiagen, Germany). In another sample, one BRA male was crossed with five virgin BRA females to create an isomale BRA line. Males from this isomale line were crossed with red-eyed Rexville females and the F1 were sib-mated. F2 red-eye females were then backcrossed with the wild-type black-eyed males from the isomale line. F3 black-eyed males were crossed with the red-eyed females originated from the F7 PAK Red-Eye GSS. Twenty red-eyed female progeny (F7PAKF3BRA_F) were used for HMW DNA extraction, as described above. Genomic DNA from F8ThaiF9Mex_F, F8ThaiF9Mex_M and F7PAKF3BRA_F were sequenced by Illumina (Supplemental Table S1).

Homozygosity analysis: Mapping and variant calling were performed as described for MAM except that samples are the hybrid sequencing samples F8ThaiF9Mex_F, F8ThaiF9Mex_M and F7PAKF3BRA_F. As the coverage is lower than those in the MAM analysis, variant filtering was adjusted based on $5<$ total read depth $<500$, only one alternative in the variant and GATK GQ score $>=50$. Within a given sliding window size (e.g., $1 \mathrm{Mb}$ or $20 \mathrm{~kb}$ ), the alternative homozygosity rate is calculated by

Alternative homozygosity rate

$$
=\frac{\text { The number of unique alternative variants showing } 100 \% \text { frequency }(\text { SNPindex }=1)}{\text { The number of all unique alternative variants (SNPindex }>0)}
$$

We focused on unique alternative SNPs as we are only interested in SNPs showing $100 \%$ frequency.

Mosquito imaging: Imaging larvae, pupae, and adult specimens were performed using an AMSCOPE LED Trinocular Zoom Stereo Microscope 3.5X-180X and 10MP USB3 Camera (https://www.amscope.com/applications/veterinary-zoology/entomology/led-trinocular-zoomstereo-microscope-3-5x-180x-and-10mp-usb3-camera.html).

CRISPR/Cas9-mediated Knockout of cardinal: For somatic knockout of cardinal, LVP males were mated with RED female mosquitoes in a $G_{-1}$ cross. The resulting $G_{0}$ embryos were injected with a mixture containing $300 \mathrm{ng} / \mathrm{uL}$ Cas9 mRNA and $100 \mathrm{ng} / \mathrm{uL}$ each of sgRNAs 1, 2, and 3. Somatic knockout of cardinal was evaluated visually at the larval pupal stages. To produce a germline knockout of cardinal, an injection mixture containing $300 \mathrm{ng} / \mathrm{uL}$ Cas $9 \mathrm{mRNA}$ and $100 \mathrm{ng} / \mathrm{uL}$ each of sgRNAs 1, 2, 4, and 5 was injected into LVP embryos. Male and female $\mathrm{G}_{0}$ adult survivors were separated and mated with RED adults of the opposite sex. Specific cardinal mutations were validated by sequencing PCR amplicons from each sgRNA target region. Briefly, genomic DNA extracted using the QiaAMP gDNA-micro kit (Qiagen) or Zymo Quick-gDNA (Zymo Research) was used as the template for PCR using primers listed in Table S4. PCR amplicons were purified using the Nucleospin PCR and gel clean-up kit (Machery-Nagel) and sequenced using Sanger (Genomics Sequencing Center, Virginia Tech). Trace files used to validate germline knockouts of cardinal are in the Supplemental File.

sgRNA design and sgRNA synthesis. sgRNAs were designed to target two regions within the predicted coding sequence of the cardinal gene (Figure 6A). The first sgRNA target region is located in exon 3 at 664-737bp from the predicted start codon and the second in exon 5 at 1,698$1,776 \mathrm{bp}$ and is near the region targeted for the recent knockout of cardinal in Culex quinquefaciatus (Feng et al. 2020). All five sgRNAs (Table S2) were designed using CHOPCHOP ((Labun et al. 2016; Labun et al. 2019), (Montague et al. 2014)) and CRISPOR (Concordet and 
Haeussler 2018) web tools. sgRNA DNA templates were synthesized by PCR using previously described protocols (Bassett et al. 2013) using primers listed in Supplemental Table S4. sgRNA was synthesized by T7 in vitro transcription using the MEGAscript T7 kit, and purified using the MEGAclear kit (Thermo Fisher Scientific) then aliquoted for embryonic microinjections (Basu et al. 2015).

\section{Acknowledgements:}

This work is supported by NIH grants R01Al123338 and 1R01Al157491 and the Virginia Agriculture Experimental Station. AC is supported by a fellowship from the Robert Wood Johnson Foundation. This study is also financially supported by the Joint FAO/IAEA Insect Pest Control Subprogramme of the Joint FAO/IAEA Centre of Nuclear Techniques in Food and Agriculture and the U.S. State Department in the frame of the 'Surge Expansion of the Sterile Insect Technique (SIT) to Control Mosquito Populations that Transmit the Zika Virus' project. This study benefitted from discussions during meetings in the frame of the IAEA Coordinated Research Project "Generic approach for the development of genetic sexing strains for SIT applications". We thank Song Li for discussions on data analysis. We thank the Chloé Lahondère laboratory for use of the Amscope microscope imaging system and for advice on imaging. We thank Margareth Capurro, Jorge Aurelio Torres Monzon, Pat Kittayapong, and Muhammad Misbah-ul-Haq for kindly providing the BRA, Mex, Thai and Pak strains used in the present study.

\section{Data deposition:}

Illumina and Oxford Nanopore sequencing data are deposited and available at https://www.ncbi.nlm.nih.gov/sra/PRJNA718905.

Table 1. Sex-linkage of the P10 (GFP) transgene insertion.

\begin{tabular}{|l|r|r|r|r|}
\hline & GFP positive male & GFP negative male & GFP positive female & GFP negative female \\
\hline$G_{2}$ & 86 & 0 & 1 & 84 \\
\hline$G_{8}$ & 743 & 12 & 9 & 589 \\
\hline $\begin{array}{l}(\mathrm{P} 10 /-;+/ r e) \times(-/-; \\
\text { re/re })\end{array}$ & 1573 & 22 & 10 & 1540 \\
\hline
\end{tabular}

Genetic distance between the sex locus $(\mathrm{M} / \mathrm{m})$ and the $\mathrm{P} 10$ insertion is calculated as follows: $(12+9) /(12+9+743+589)=1.55 \%=1.55 \mathrm{cM}$, according to the $\mathrm{G}_{8}$ data and $(22+10) /(22+10+1573+1540)=1.02 \%=1.02 \mathrm{cM}$, according to the cross shown in the last row. The numbers are too low in $\mathrm{G}_{2}$ for an accurate estimation.

\section{References}

Aryan, A., M. A. E. Anderson, J. K. Biedler, Y. Qi, J. M. Overcash, A. N. Naumenko, M. V. Sharakhova, C. Mao, Z. N. Adelman, and Z. Tu. 2020. 'Nix alone is sufficient to convert female Aedes aegypti into fertile males and myo-sex is needed for male flight', Proc Natl Acad Sci U S A, 117: 17702-09. 
Augustinos, A. A., M. Misbah-Ul-Haq, D. O. Carvalho, L. D. de la Fuente, P. Koskinioti, and K. Bourtzis. 2020. 'Irradiation induced inversions suppress recombination between the M locus and morphological markers in Aedes aegypti', BMC Genet, 21: 142.

Balatsos, G., Puggioli, A., Karras, V., Lytra, I,, Mastronikolos, G., Carrieri, M., Papachristos, D.P., Malfacini, M., Stefopoulou, A., loannou, C.S., Balestrino, F., Bouyer, J., Petrić, D., Pajović, I., Kapranas, A., Papadopoulos, N.T., Milonas, P.G., Bellini, R., and A.

Michaelakis. 2021. Reduction in Egg Fertility of Aedes albopictus Mosquitoes in Greece Following Releases of Imported Sterile Males. Insects. 12:110.

Bassett, A. R., C. Tibbit, C. P. Ponting, and J. L. Liu. 2013. 'Highly efficient targeted mutagenesis of Drosophila with the CRISPR/Cas9 system', Cell Rep, 4: 220-8.

Basu, S., A. Aryan, J. M. Overcash, G. H. Samuel, M. A. Anderson, T. J. Dahlem, K. M. Myles, and Z. N. Adelman. 2015. 'Silencing of end-joining repair for efficient site-specific gene insertion after TALEN/CRISPR mutagenesis in Aedes aegypti', Proc Natl Acad Sci U S A, 112: 4038-43.

Bolger, A. M., M. Lohse, and B. Usadel. 2014. 'Trimmomatic: a flexible trimmer for Illumina sequence data', Bioinformatics, 30: 2114-20.

Coates, C. J., N. Jasinskiene, L. Miyashiro, and A. A. James. 1998. 'Mariner transposition and transformation of the yellow fever mosquito, Aedes aegypti', Proc Natl Acad Sci U S A, 95: 3748-51.

Concordet, J. P., and M. Haeussler. 2018. 'CRISPOR: intuitive guide selection for CRISPR/Cas9 genome editing experiments and screens', Nucleic Acids Res, 46: W242W45.

Craig, G. B., Jr., and W. A. Hickey. 1967. 'Current status of the formal genetics of Aedes aegypti', Bull World Health Organ, 36: 559-62.

Crawford, J. E., D. W. Clarke, V. Criswell, M. Desnoyer, D. Cornel, B. Deegan, K. Gong, K. C. Hopkins, P. Howell, J. S. Hyde, J. Livni, C. Behling, R. Benza, W. Chen, K. L. Dobson, C. Eldershaw, D. Greeley, Y. Han, B. Hughes, E. Kakani, J. Karbowski, A. Kitchell, E. Lee, T. Lin, J. Liu, M. Lozano, W. MacDonald, J. W. Mains, M. Metlitz, S. N. Mitchell, D. Moore, J. R. Ohm, K. Parkes, A. Porshnikoff, C. Robuck, M. Sheridan, R. Sobecki, P. Smith, J. Stevenson, J. Sullivan, B. Wasson, A. M. Weakley, M. Wilhelm, J. Won, A. Yasunaga, W. C. Chan, J. Holeman, N. Snoad, L. Upson, T. Zha, S. L. Dobson, F. S. Mulligan, P. Massaro, and B. J. White. 2020. 'Efficient production of male Wolbachiainfected Aedes aegypti mosquitoes enables large-scale suppression of wild populations', Nat Biotechnol, 38: 482-92.

Curtis, C.F. 1978. Genetic sex separation in Anopheles arabiensis and the production of sterile hybrids. Bull World Health Organ. 56: 453-454.

DePristo, M. A., E. Banks, R. Poplin, K. V. Garimella, J. R. Maguire, C. Hartl, A. A. Philippakis, G. del Angel, M. A. Rivas, M. Hanna, A. McKenna, T. J. Fennell, A. M. Kernytsky, A. Y. Sivachenko, K. Cibulskis, S. B. Gabriel, D. Altshuler, and M. J. Daly. 2011. 'A framework for variation discovery and genotyping using next-generation DNA sequencing data', Nat Genet, 43: 491-8.

Ding, Y., A. Berrocal, T. Morita, K. D. Longden, and D. L. Stern. 2016. 'Natural courtship song variation caused by an intronic retroelement in an ion channel gene', Nature, 536: 32932.

Dudchenko, O., S. S. Batra, A. D. Omer, S. K. Nyquist, M. Hoeger, N. C. Durand, M. S. Shamim, I. Machol, E. S. Lander, A. P. Aiden, and E. L. Aiden. 2017. 'De novo assembly of the Aedes aegypti genome using $\mathrm{Hi}-\mathrm{C}$ yields chromosome-length scaffolds', Science, 356: 92-95.

Feng, J., X. Zhang, M. Zhang, L. Guo, T. Qi, H. Tang, H. Zhu, H. Wang, X. Qiao, C. Xing, and J. Wu. 2021. 'Physical mapping and InDel marker development for the restorer gene Rf2 in cytoplasmic male sterile CMS-D8 cotton', BMC Genomics, 22: 24. 
Feng, Xuechun, Lukas Kambic, Jared HK Nishimoto, Floyd A Reed, Jai A Denton, Jolene T Sutton, and Valentino M Gantz. 2020. 'Evaluation of pigmentation gene knock-outs by CRISPR as potential targets for the genetic engineering of Culex quinquefasciatus', bioRxiv.

Fontaine, A., I. Filipovic, T. Fansiri, A. A. Hoffmann, C. Cheng, M. Kirkpatrick, G. Rasic, and L. Lambrechts. 2017. 'Extensive Genetic Differentiation between Homomorphic Sex Chromosomes in the Mosquito Vector, Aedes aegypti', Genome Biol Evol, 9: 2322-35.

Franz, G., Bourtzis, K. and C. Cáceres. 2021. Practical and operational genetic sexing systems based on classical genetic approaches in fruit flies, an example for other species amenable to large-scale rearing for the sterile insect technique. In: V. A. Dyck, J. Hendrichs and A. S. Robinson (eds.), Sterile Insect Technique. Principles and Practice in Area-Wide Integrated Pest Management. Second Edition. CRC Press, Boca Raton, Florida, USA, pp 575-604.

Hall, Andrew Brantley, Sanjay Basu, Xiaofang Jiang, Yumin Qi, Vladimir A Timoshevskiy, James K Biedler, Maria V Sharakhova, Rubayet Elahi, Michelle AE Anderson, and XiaoGuang Chen. 2015. 'A male-determining factor in the mosquito Aedes aegypti', Science, 348: 1268-70.

Handler, A. M., S. D. McCombs, M. J. Fraser, and S. H. Saul. 1998. 'The lepidopteran transposon vector, piggyBac, mediates germ-line transformation in the Mediterranean fruit fly', Proc Natl Acad Sci U S A, 95: 7520-5.

Harris, D. A., K. Kim, K. Nakahara, C. Vasquez-Doorman, and R. W. Carthew. 2011. 'Cargo sorting to lysosome-related organelles regulates siRNA-mediated gene silencing', J Cell Biol, 194: 77-87.

Howells, A. J., K. M. Summers, and R. L. Ryall. 1977. 'Developmental patterns of 3hydroxykynurenine accumulation in white and various other eye color mutants of Drosophila melanogaster', Biochem Genet, 15: 1049-59.

Juneja, P., J. Osei-Poku, Y. S. Ho, C. V. Ariani, W. J. Palmer, A. Pain, and F. M. Jiggins. 2014. 'Assembly of the genome of the disease vector Aedes aegypti onto a genetic linkage map allows mapping of genes affecting disease transmission', PLoS Negl Trop Dis, 8: e2652.

Kaiser, P.E., Seawright, J., Dame, D.A., and D. J. Joslyn. 1978. Development of a Genetic Sexing System for Anopheles albimanus, Journal of Economic Entomology, 71: 766771.

Kistler, K. E., L. B. Vosshall, and B. J. Matthews. 2015. 'Genome engineering with CRISPRCas9 in the mosquito Aedes aegypti', Cell Rep, 11: 51-60.

Kittayapong, P., Ninphanomchai, S., Limohpasmanee, W., Chansang, C., Chansang, U., and P. Mongkalangoon. 2019. Combined sterile insect technique and incompatible insect technique: The first proof-of-concept to suppress Aedes aegypti vector populations in semi-rural settings in Thailand. PLoS Negl Trop Dis., 13: e0007771.

Koskinioti, P., A. A. Augustinos, D. O. Carvalho, M. Misbah-Ul-Haq, G. Pillwax, L. D. de la Fuente, G. Salvador-Herranz, R. A. Herrero, and K. Bourtzis. 2021. 'Genetic sexing strains for the population suppression of the mosquito vector Aedes aegypti', Philos Trans $R$ Soc Lond B Biol Sci, 376: 20190808.

Labun, K., T. G. Montague, J. A. Gagnon, S. B. Thyme, and E. Valen. 2016. 'CHOPCHOP v2: a web tool for the next generation of CRISPR genome engineering', Nucleic Acids Res, 44: W272-6.

Labun, K., T. G. Montague, M. Krause, Y. N. Torres Cleuren, H. Tjeldnes, and E. Valen. 2019. 'CHOPCHOP v3: expanding the CRISPR web toolbox beyond genome editing', Nucleic Acids Res, 47: W171-W74.

$\mathrm{Li}, \mathrm{H}$., and R. Durbin. 2009. 'Fast and accurate short read alignment with Burrows-Wheeler transform', Bioinformatics, 25: 1754-60. 
Li, H., B. Handsaker, A. Wysoker, T. Fennell, J. Ruan, N. Homer, G. Marth, G. Abecasis, R. Durbin, and Subgroup Genome Project Data Processing. 2009. 'The Sequence Alignment/Map format and SAMtools', Bioinformatics, 25: 2078-9.

Li, M., M. Bui, T. Yang, C. S. Bowman, B. J. White, and O. S. Akbari. 2017. 'Germline Cas9 expression yields highly efficient genome engineering in a major worldwide disease vector, Aedes aegypti', Proc Natl Acad Sci U S A, 114: E10540-E49.

Lines, J.D. and C. F. Curtis 1985. Genetic Sexing Systems in Anopheles arabiensis Patton (Diptera: Culicidae), Journal of Economic Entomology, 78: 848-851.

Mansfeld, B.N. and R. Grumet. 2018. QTLseqr: An R Package for Bulk Segregant Analysis with Next-Generation Sequencing. The Plant Genome, 11: 10.3835.

Matthews, B. J., O. Dudchenko, S. B. Kingan, S. Koren, I. Antoshechkin, J. E. Crawford, W. J. Glassford, M. Herre, S. N. Redmond, N. H. Rose, G. D. Weedall, Y. Wu, S. S. Batra, C. A. Brito-Sierra, S. D. Buckingham, C. L. Campbell, S. Chan, E. Cox, B. R. Evans, T. Fansiri, I. Filipovic, A. Fontaine, A. Gloria-Soria, R. Hall, V. S. Joardar, A. K. Jones, R. G. G. Kay, V. K. Kodali, J. Lee, G. J. Lycett, S. N. Mitchell, J. Muehling, M. R. Murphy, A. D. Omer, F. A. Partridge, P. Peluso, A. P. Aiden, V. Ramasamy, G. Rasic, S. Roy, K. Saavedra-Rodriguez, S. Sharan, A. Sharma, M. L. Smith, J. Turner, A. M. Weakley, Z. Zhao, O. S. Akbari, W. C. th Black, H. Cao, A. C. Darby, C. A. Hill, J. S. Johnston, T. D. Murphy, A. S. Raikhel, D. B. Sattelle, I. V. Sharakhov, B. J. White, L. Zhao, E. L. Aiden, R. S. Mann, L. Lambrechts, J. R. Powell, M. V. Sharakhova, Z. Tu, H. M. Robertson, C. S. McBride, A. R. Hastie, J. Korlach, D. E. Neafsey, A. M. Phillippy, and L. B. Vosshall. 2018. 'Improved reference genome of Aedes aegypti informs arbovirus vector control', Nature, 563: 501-07.

McClelland, G. A. 1966. 'Sex-linkage at two loci affecting eye pigment in the mosquito Aedes aegypti (diptera: culicidae)', Can J Genet Cytol, 8: 192-8.

McClelland, GAH. 1962. 'Sex-linkage in Aedes aegypti', Trans roy Soc trop Med Hyg, 56.

McKenna, A., M. Hanna, E. Banks, A. Sivachenko, K. Cibulskis, A. Kernytsky, K. Garimella, D. Altshuler, S. Gabriel, M. Daly, and M. A. DePristo. 2010. 'The Genome Analysis Toolkit: a MapReduce framework for analyzing next-generation DNA sequencing data', Genome Res, 20: 1297-303.

Montague, T. G., J. M. Cruz, J. A. Gagnon, G. M. Church, and E. Valen. 2014. 'CHOPCHOP: a CRISPR/Cas9 and TALEN web tool for genome editing', Nucleic Acids Res, 42: W401-7.

Mysore, K., P. Li, and M. Duman-Scheel. 2018. 'Identification of Aedes aegypti cis-regulatory elements that promote gene expression in olfactory receptor neurons of distantly related dipteran insects', Parasit Vectors, 11: 406.

Navarro-Escalante, L., C. Zhao, R. Shukle, and J. Stuart. 2020. 'BSA-Seq Discovery and Functional Analysis of Candidate Hessian Fly (Mayetiola destructor) Avirulence Genes', Front Plant Sci, 11: 956.

Osanai-Futahashi, M., K. I. Tatematsu, R. Futahashi, J. Narukawa, Y. Takasu, T. Kayukawa, T. Shinoda, T. Ishige, S. Yajima, T. Tamura, K. Yamamoto, and H. Sezutsu. 2016. 'Positional cloning of a Bombyx pink-eyed white egg locus reveals the major role of cardinal in ommochrome synthesis', Heredity (Edinb), 116: 135-45.

Papathanos, P. A., K. Bourtzis, F. Tripet, H. Bossin, J. F. Virginio, M. L. Capurro, M. C. Pedrosa, A. Guindo, L. Sylla, M. B. Coulibaly, F. A. Yao, P. S. Epopa, and A. Diabate. 2018. 'A perspective on the need and current status of efficient sex separation methods for mosquito genetic control', Parasit Vectors, 11: 654.

Robinson A. S. 1986. Genetic sexing in Anopheles stephensi using dieldrin resistance. Journal of the American Mosquito Control Association, 2: 93-95.

Rose, N. H., M. Sylla, A. Badolo, J. Lutomiah, D. Ayala, O. B. Aribodor, N. Ibe, J. Akorli, S. Otoo, J. P. Mutebi, A. L. Kriete, E. G. Ewing, R. Sang, A. Gloria-Soria, J. R. Powell, R. E. 
Baker, B. J. White, J. E. Crawford, and C. S. McBride. 2020. 'Climate and Urbanization Drive Mosquito Preference for Humans', Curr Biol, 30: 3570-79 e6.

Schneeberger, K. 2014. 'Using next-generation sequencing to isolate mutant genes from forward genetic screens', Nat Rev Genet, 15: 662-76.

Stapley, J., P. G. D. Feulner, S. E. Johnston, A. W. Santure, and C. M. Smadja. 2017a. 'Recombination: the good, the bad and the variable', Philos Trans $R$ Soc Lond B Biol Sci, 372.

Stapley, J., P. G. D. Feulner, S. E. Johnston, A. W. Santure, and C. M. Smadja. 2017b. 'Variation in recombination frequency and distribution across eukaryotes: patterns and processes', Philos Trans R Soc Lond B Biol Sci, 372.

Takagi, H., Abe, A., Yoshida, K., Kosugi, S., Natsume, S., Mitsuoka, C., Uemura, A., Utsushi, H., Tamiru, M., Takuno, S., Innan, H., Cano, L. M., Kamoun, S. and R. Terauchi. 2013. QTL-seq: rapid mapping of quantitative trait loci in rice by whole genome resequencing of DNA from two bulked populations. The Plant journal, 74: 174-183. https://doi.org/10.1111/tpj.12105

Van der Auwera, G. A., M. O. Carneiro, C. Hartl, R. Poplin, G. Del Angel, A. Levy-Moonshine, T. Jordan, K. Shakir, D. Roazen, J. Thibault, E. Banks, K. V. Garimella, D. Altshuler, S. Gabriel, and M. A. DePristo. 2013. 'From FastQ data to high confidence variant calls: the Genome Analysis Toolkit best practices pipeline', Curr Protoc Bioinformatics, 43: 1110 1-11 1033.

Ward, C. M., R. A. Aumann, M. A. Whitehead, K. Nikolouli, G. Leveque, G. Gouvi, E. Fung, S. J. Reiling, H. Djambazian, M. A. Hughes, S. Whiteford, C. Caceres-Barrios, T. N. M. Nguyen, A. Choo, P. Crisp, S. B. Sim, S. M. Geib, F. Marec, I. Hacker, J. Ragoussis, A. C. Darby, K. Bourtzis, S. W. Baxter, and M. F. Schetelig. 2021. 'White pupae phenotype of tephritids is caused by parallel mutations of a MFS transporter', Nat Commun, 12: 491.

Wilfert, L., J. Gadau, and P. Schmid-Hempel. 2007. 'Variation in genomic recombination rates among animal taxa and the case of social insects', Heredity (Edinb), 98: 189-97.

Xu, X., T. Harvey-Samuel, J. Yang, L. Alphey, and M. You. 2020. 'Ommochrome pathway genes kynurenine 3-hydroxylase and cardinal participate in eye pigmentation in Plutella xylostella', BMC Mol Cell Biol, 21: 63.

Yamada, H., Benedict, M. Q., Malcolm, C. A., Oliva, C. F., Soliban, S. M., and J.R. Gilles. 2012. Genetic sex separation of the malaria vector, Anopheles arabiensis, by exposing eggs to dieldrin. Malaria journal, 11: 208.

Yamada, H., Vreysen, M. J., Bourtzis, K., Tschirk, W., Chadee, D. D., and J.R. Gilles. 2015. The Anopheles arabiensis genetic sexing strain ANO IPCL1 and its application potential for the sterile insect technique in integrated vector management programmes. Acta tropica, 142: 138-144.

Zhang, H., Y. Lin, G. Shen, X. Tan, C. Lei, W. Long, H. Liu, Y. Zhang, Y. Xu, J. Wu, J. Gu, Q. Xia, and P. Zhao. 2017. 'Pigmentary analysis of eggs of the silkworm Bombyx mori', $J$ Insect Physiol, 101: 142-50.

Zheng, X., D. Zhang, Y. Li, C. Yang, Y. Wu, X. Liang, Y. Liang, X. Pan, L. Hu, Q. Sun, X. Wang, Y. Wei, J. Zhu, W. Qian, Z. Yan, A. G. Parker, J. R. L. Gilles, K. Bourtzis, J. Bouyer, M. Tang, B. Zheng, J. Yu, J. Liu, J. Zhuang, Z. Hu, M. Zhang, J. T. Gong, X. Y. Hong, Z. Zhang, L. Lin, Q. Liu, Z. Hu, Z. Wu, L. A. Baton, A. A. Hoffmann, and Z. Xi. 2019. 'Incompatible and sterile insect techniques combined eliminate mosquitoes', Nature, 572: 56-61. 

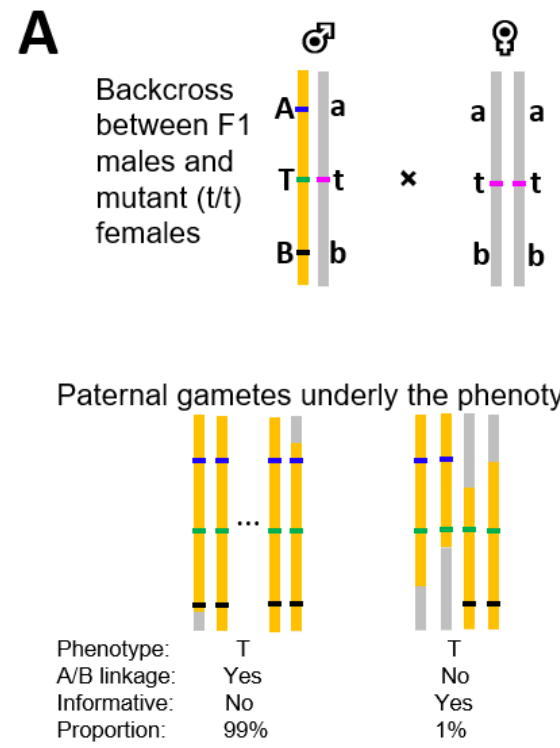
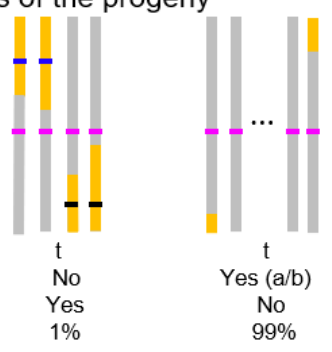

B

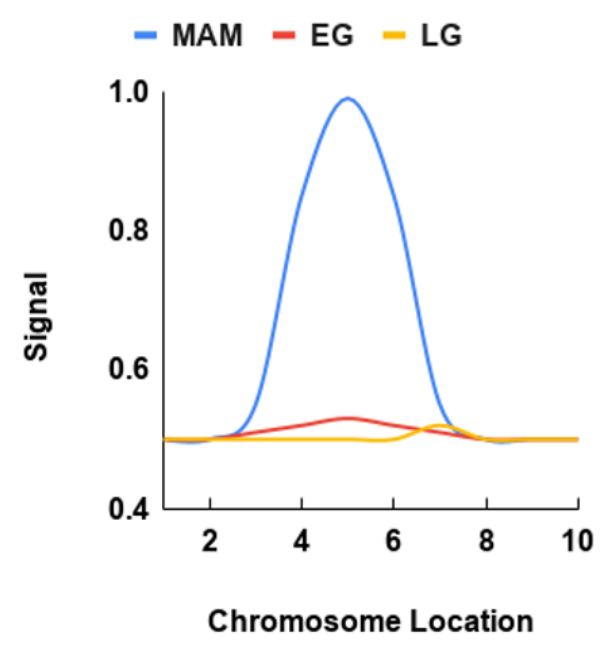

Figure 1. The marker-assisted mapping (MAM) strategy. A) The progeny of a backcross between F1 males and mutant $(t / t)$ females can be categorized according to the mutant phenotype (T/t versus $t / t)$ and the linkage between two easy-to-screen flanking markers (A and $B)$. Only paternal gamete is shown. In the example shown here, the genetic distance between $A$ and $B$ is $1 \mathrm{cM}$. Thus, $99 \%$ of the progeny that retain the linkage between the $A$ and $B$ markers are not informative and can be ignored. B). Expected signal strengths using either marker-assisted mapping (MAM) when only the informative progeny (T/t and $t / t$ progeny showing breakage between markers $A$ and $B)$, extreme genotyping (EG) when hundreds of $T / t$ and $t / t$ progeny are analyzed, or limited genotyping (LG) when a limited sample of the $T / t$ and $t / t$ progeny are genotyped. In the current example, genotyping $100 \mathrm{~T} / \mathrm{t}$ and $100 \mathrm{t} / \mathrm{t}$ progeny will likely only include 1 informative $\mathrm{T} / \mathrm{t}$ and 1 informative $\mathrm{t} / \mathrm{t}$ recombinant between the flanking markers, severely limiting the signal to noise ratio and the resolution of the mapping result. The position of the "peak" in LG could also be misleading due to limited sampling. 


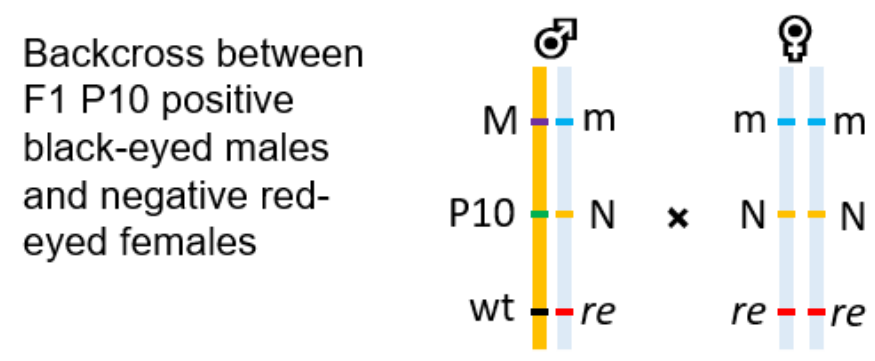

Paternal gametes underly the phenotypes of the progeny

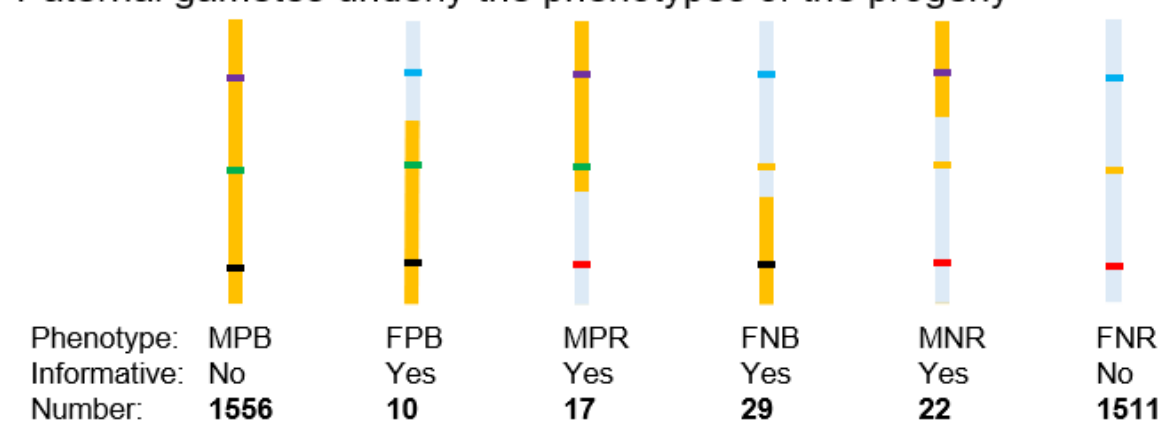

Figure 2. Marker-assisted mapping of the P10 transgene insertion. An F1 backcross and the resulting F2 progeny. Double crossovers, which are negligible in this case, are not shown. M: the dominant maledetermining locus; $\mathrm{M} / \mathrm{m}$ are at $152 \mathrm{Mb}$ of chromosome 1. P10: positive for the P10 GFP transgene insertion; $\mathrm{N}$ : negative or no transgene insertion; re: the recessive red-eye allele; wt: wildtype black-eye allele. To denote the phenotype, $M$ is male while $F$ is female; $P$ is $P 10$ transgene positive while $N$ is negative; $B$ is black-eyed while R is red-eyed. For example, MPB is a male P10 positive black-eyed individual. While crossover could potentially happen anywhere between markers, only one example is provided. The answer regarding to informative or not is in the context of mapping $\mathrm{P} 10$. The genetic distance between $\mathrm{P} 10$ and the $\mathrm{M} / \mathrm{m}$ loci is $(10+22) /(1556+10+17+29+22+1511)=32 / 3145=1.02 \%$, or $1.02 \mathrm{cM}$. The genetic distance between $\mathrm{P} 10$ and $r e$ is $(17+29) / 3145=1.46 \%$, or $1.46 \mathrm{cM}$. The genetic distance between re and the $\mathrm{M} / \mathrm{m}$ locus is $(10+22+17+29) / 3145=2.48 \%$, or $2.48 \mathrm{cM}$. 


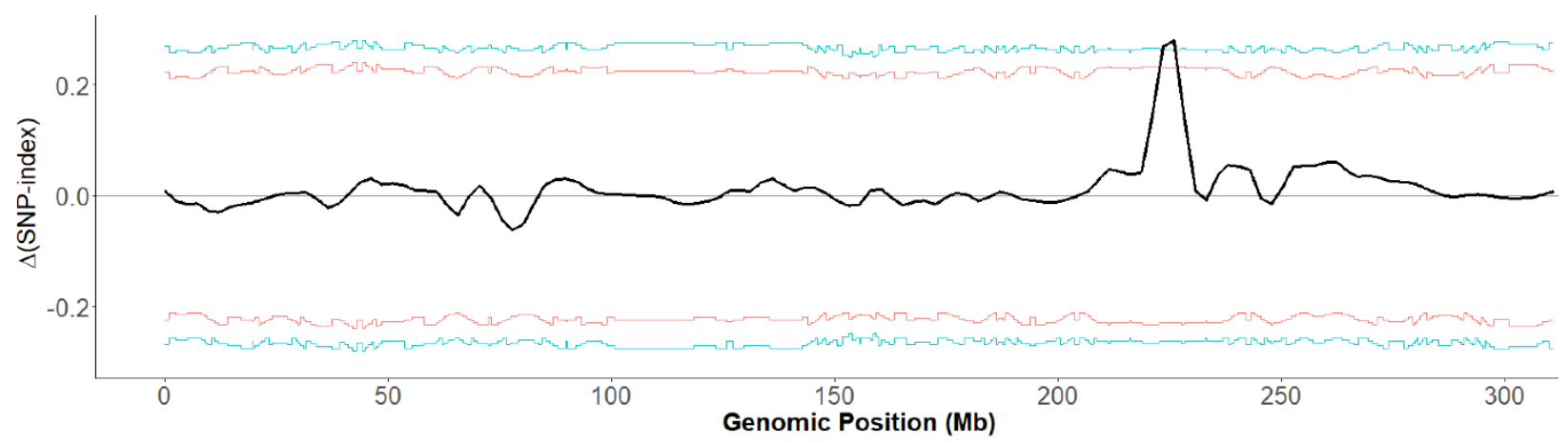

Figure 3. Marker-assisted mapping (MAM) identifies the location of the P10 transgene insertion. The tricube-smoothed $\Delta$ (SNP-index) is shown in $1 \mathrm{Mb}$ sliding windows. The $\Delta$ (SNP-index) value is calculated by comparing all informative P10 positive recombinants (MPR and FPB) with all informative P10 negative recombinants (FNB and MNR). The corresponding two-sided confidence intervals are shown as red $(90 \%)$ or blue (95\%) lines. X-axis is the genomic position on the homomorphic sex chromosome 1. The confidence intervals were estimated using 10,000 replicate simulations for all SNP positions with the given read depths. P10 is located at position $224,678,447$ on chromosome 1 , according to ONT sequencing (Supplemental Figure S3). Positions from 223288153 to 226114389 exceeds 95\% confidence and the left and right inflection points of this peak are $223412798-225833455$, respectively. 

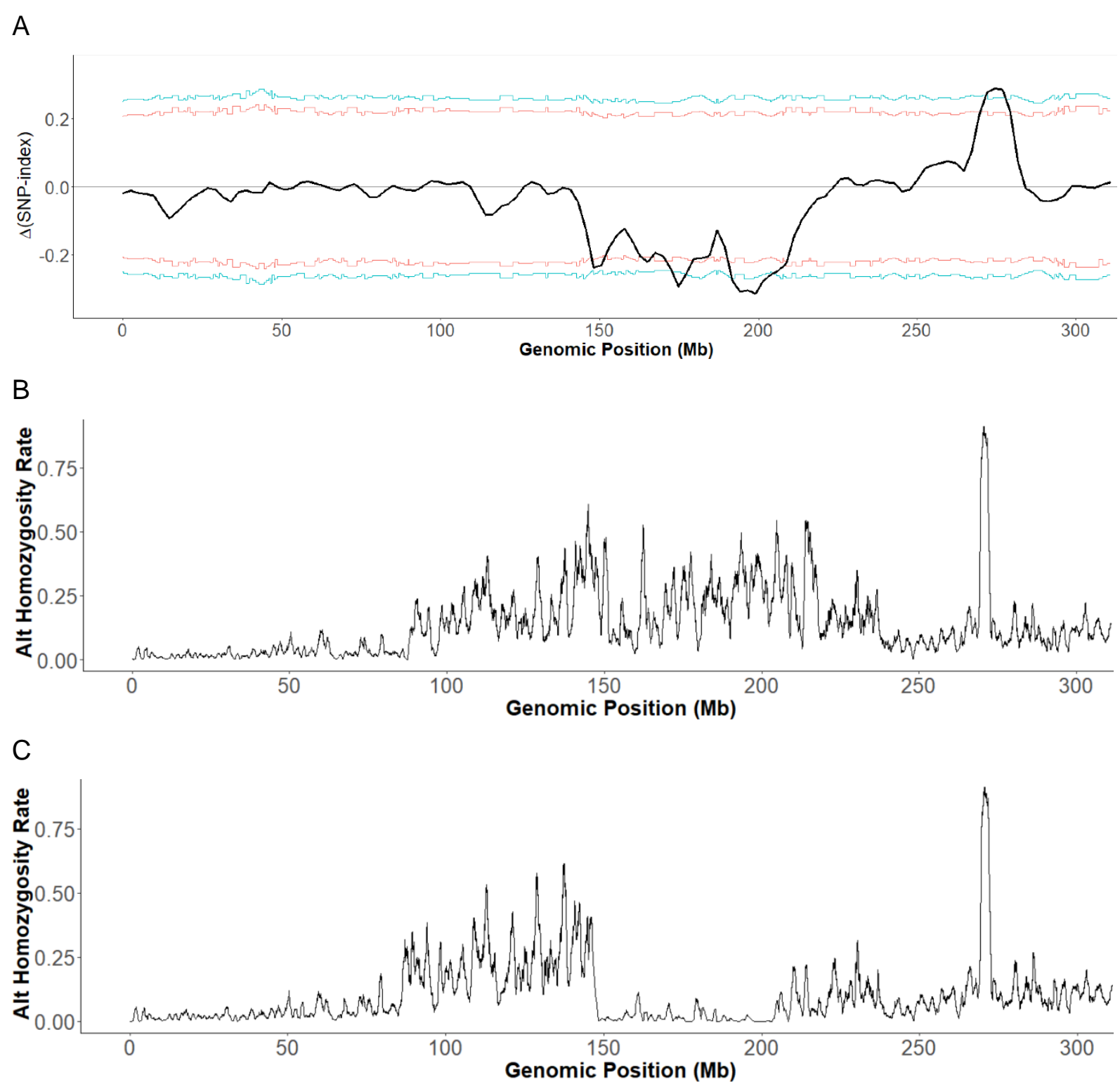

$\mathrm{D}$

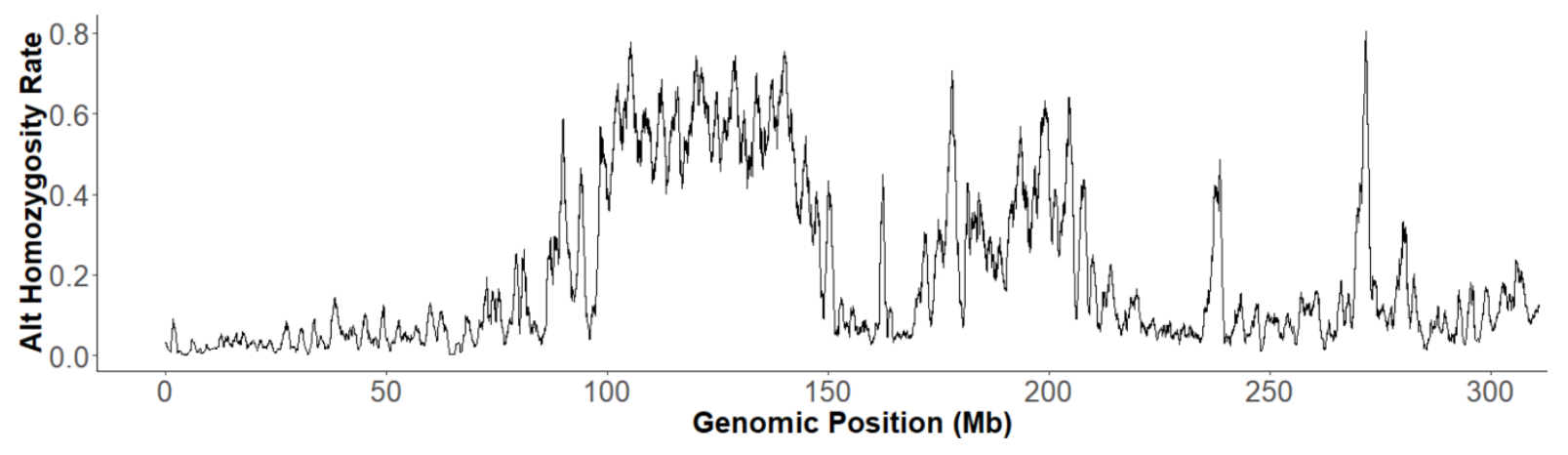

Figure 4. Mapping of re. A) Marker-assisted mapping (MAM) of re by plotting the $\Delta$ (SNP-index) values in $1 \mathrm{Mb}$ sliding windows. The corresponding two-sided confidence intervals are shown as 
red $(90 \%)$ or blue $(95 \%)$ lines. X-axis is the genomic position on the homomorphic sex chromosome 1 . The confidence intervals were estimated using 10,000 replicate simulations for all the SNP positions with the given read depths. The broad depression between 150-210 Mb likely reflects the sex-linked differences while the peak around $275 \mathrm{Mb}$ is likely the location of $r e$. Positions from $271,120,117$ to $277,843,549$ exceeds $95 \%$ confidence and the left and right inflection points of this peak are $271,970,355$ and $276,823,052$, respectively. B)-D). Homozygosity plots of red-eyed hybrids from F8ThaiF9Mex_F (B), F8ThaiF9Mex_M (C) and F7PAKF3BRA_F (D). $\mathrm{X}$-axis is the genomic location on chromosome 1. Y-axis is the homozygosity of the alternative or non-reference alleles, which is calculated as (the number of unique alternative SNPs showing $100 \%$ frequency)/(the number of all unique alternative SNPs) in a $1 \mathrm{Mb}$ window. We focused on unique alternative SNPs as we are only interested in SNPs showing 100\% frequency. The peak for F8ThaiF9Mex_F (B), F8ThaiF9Mex_M (C) and F7PAKF3BRA_F (D) are 270-271 Mb, 270$271 \mathrm{Mb}$, and $271-272 \mathrm{Mb}$, respectively.

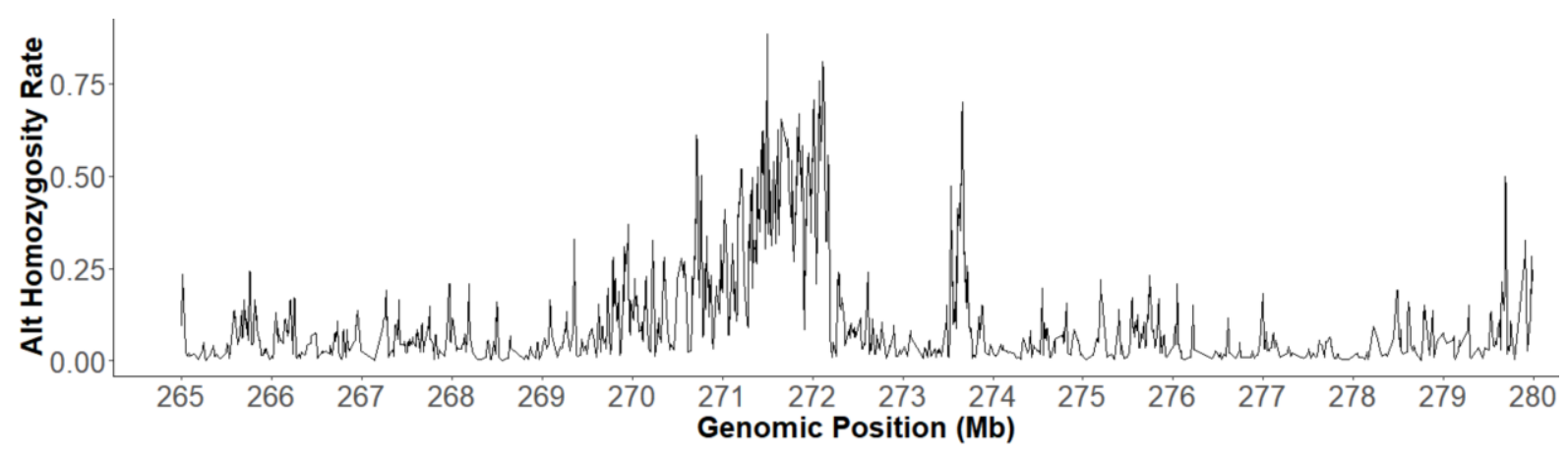

Figure 5. Homozygosity analysis between $265 \mathrm{Mbp}$ and $280 \mathrm{Mbp}$ on chromosome 1 by pooling all three hybrid red-eyed samples. Y-axis is the homozygosity of the alternative or non-reference alleles, which is calculated as (the number of unique alternative SNPs showing 100\% frequency)/(the number of all unique alternative SNPs) in a $20 \mathrm{~kb}$ window. The highest level of homozygosity is at position $271,505,401$. 


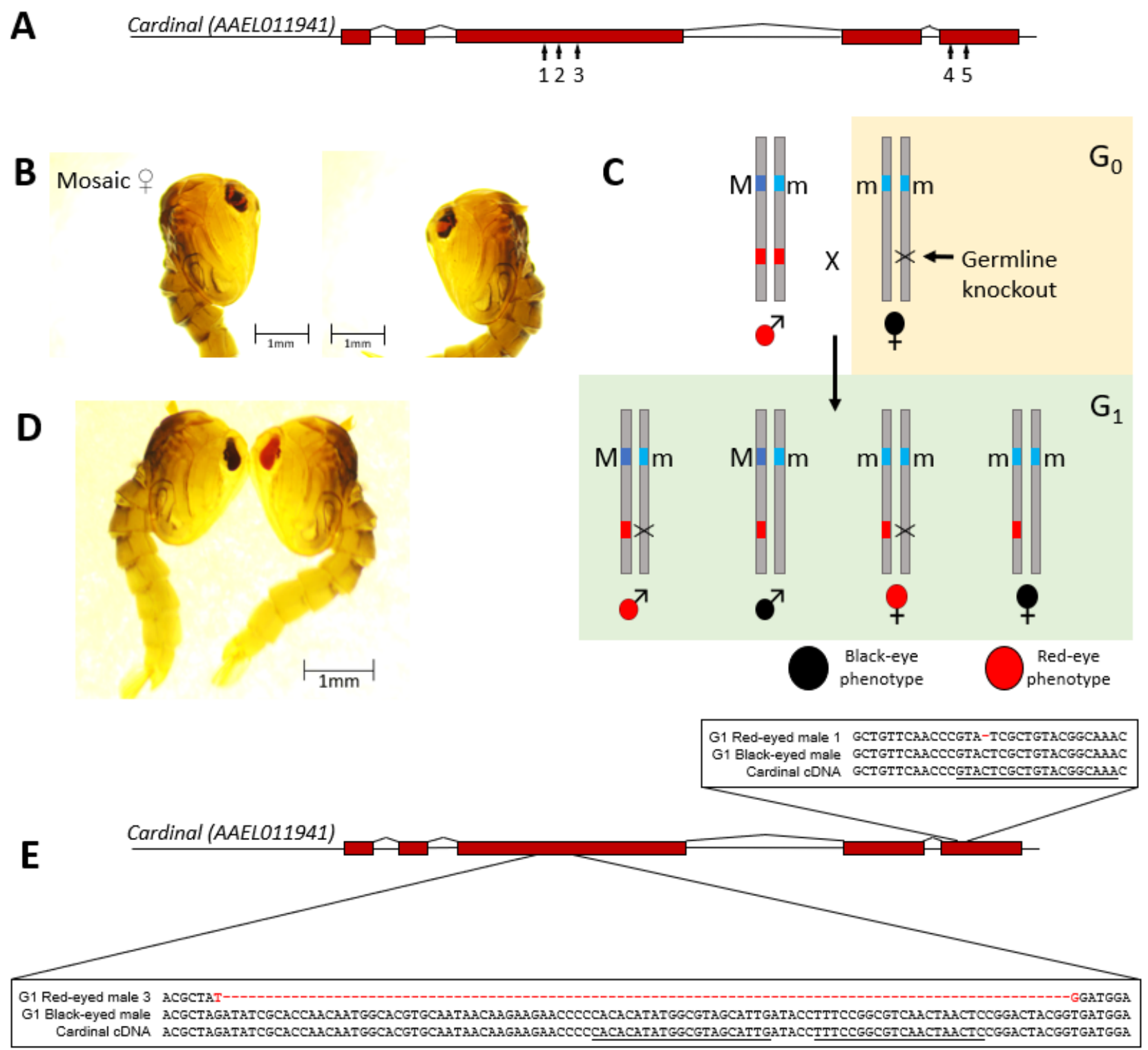

Figure 6. A) Illustration of the cardinal gene and the relative positions of the 5 sgRNAs. B) Somatic knockout of cardinal in a heterozygous (+/re) female results in a mosaic eye. Cas 9 mRNA and cardinal sgRNAs were injected into eggs that were collected from red-eyed females (re/re) mated with wildtype Liverpool strain males $(+/+)$. The two images are the right and left eyes of the same female. Images for the control blackeyed siblings and a similar mosaic-eyed male individual are shown in Supplemental Figure S6. C) Design for germline knockout of cardinal. D) $\mathrm{G}_{1}$ black-eye (left) and red-eye (right) phenotype. Four red-eyed $\mathrm{G}_{1}$ females and seven red-eyed $G_{1}$ males were identified among approximately 500 black-eyed $G_{1}$ progeny. E) Sequences show a $1 \mathrm{bp}$ deletion (in red) in red-eyed $\mathrm{G}_{1}$ male \#1 and a $99 \mathrm{bp}$ deletion (in red) in red-eyed $\mathrm{G}_{1}$ male \#3. The $1 \mathrm{bp}$ deletion was found in the other sequenced red-eyed $\mathrm{G}_{1}$ individuals (male \#1, 5, 6 and 7 ). None of the deletions was found in black-eyed $\mathrm{G}_{1}$ individuals. The sgRNA sequences near the deletion sites are underlined. 

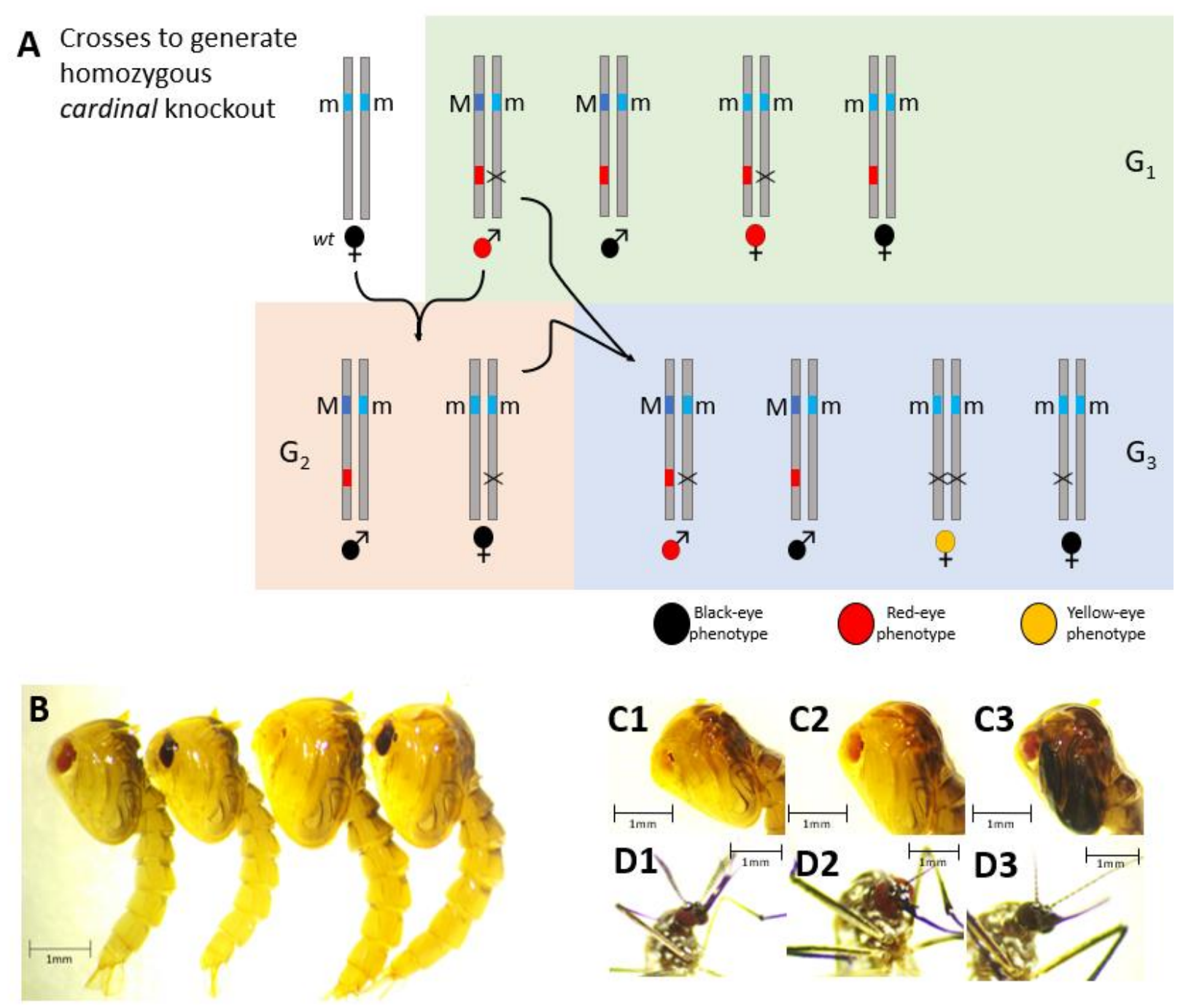

Figure 7. A) Crossing scheme to generate homozygous cardinal knockout. B) Four phenotypes observed in the G3 progeny from a cross shown in panel A. From left-to-right: red-eyed male, black-eyed male, colorless-eyed female, black-eyed female. The $\mathrm{G}_{1}$ red-eye male has the $99 \mathrm{bp}$ deletion ( $\mathrm{G}_{1}$ red-eye male\#3). C) The eyes in the colorless-eyed female turn red over the course of pupal development. C1-3: 1, 2, and 3 day old pupae. To highlight the eye phenotype, the contrast was adjusted to $20 \%$ and the brightness was adjusted to $20 \%$ (for $\mathrm{C} 1-2$ ) and $40 \%$ (C3). D) 1-day-old $\mathrm{G}_{3}$ adult eye phenotypes of the red-eyed male (D1), yellow-turn-red-eyed female (D2), and black-eyed female (D3). To highlight the eye phenotypes, the contrast of images was adjusted to $25 \%$ and the brightness to $25 \%$ (D1-2) and $50 \%$ (D3). All unmodified photos can be found in the Supplemental images file. 